\title{
Role of Noncontrast Conventional Magnetic Resonance Imaging at 3 Tesla in Evaluation of Diabetes-Related Pedal Osteomyelitis in Cases of Renal Impairment
}

A.F.Yousof, N.A.EL-Toukhy and Y.Z.karkash

Interventional radiology Dept., Faculty of Medicine, Benha Univ., Benha, Egypt

E-Mail:Y.karkash@gmail.com

\begin{abstract}
Diabetic foot contamination remains a real reason for horribleness and mortal sin in a significant number patients and stays a testing finding for A large portion clinicians. One-fifth for patients with diabetes who have foot ulcerations will create osteomyelitis. The vast majority situations of diabetic foot osteomyelitis aftereffect starting with those spread of a foot contamination on underlying bone. The point of this consider will be should assess the utilization from claiming Non-contrast T1 mri in characterization of diabetes related pedal osteomyelitis done situations for renal disappointment What's more correspondence with clinical Furthermore surgical result. Retrospective, non-controlled non-randomized consider. Utilizing 3 tesla siemens mri machine, non-contrast contemplate for T1, T2 Also T2 blend. Determination of the marrow indicator power over T1weighted pictures (preserved alternately lost) In those webpage for ulcer Assuming that available. If passing about indicator power Previously, T1: determination of the dissemination (subcortical alternately medullary), Furthermore example for association (hazy reticulated or confluent). 18 (45\%) about cases, 4th toe demonstrated the any rate influenced webpage Eventually Tom's perusing 4(10\%) Of instances. Best 2(5\%) situations were quell Eventually Tom's perusing sub cortical conveyance happened In calcaneus for reticular example. The remaining might have been spoke to Toward medullary dissemination. 4 situations were spoken to by intersecting design ( 2 In enormous toe Also 2 toward calcaneus) same time those remaining situations about cloudy example. Those number What's more rate of destinations about negative clinical result for conclusion, our study, which accentuated imaging offers ahead T1weighted pictures over surgically turned out cases about pedal osteomyelitis, indicated that immediate essential discoveries of diminished $\mathrm{T} 1$ marrow indicator On a geographic medullary dissemination with An intersecting design Furthermore concordance for the T2 marrow sign abnormality were introduce over $100 \%$ of true-positive instances. No instances from claiming surgically affirmed osteomyelitis needed elementary $\mathrm{T} 1$ discoveries for subcortical distribution; hazy, reticulated pattern; alternately harshness with the T2-weighted pictures.
\end{abstract}

Keywords: Diabetic, Non-contrast, MRI, Medullary, Retrospective, T2-weighted images.

\section{Introduction}

The ACR (American school about Radiology) gives propriety criteria for imaging for diabetic patients with suspected osteomyelitis. Radiographs remain the recommended beginning screening contemplate notwithstanding low affectability particularly with nonattendance for cortical bone decimation $(43 \%$ to $75 \%$ ) What's more specificities about $69 \%$ should $83 \%$. On the other hand, the radionuclide filters need helter skelter affectability in any case low specificity. Later investigations show a affectability from claiming 95\% Furthermore specificity for best $33 \%$ to three period bone filters [1].

Attractive reverberation imaging (MRI) is those modality about decision to imaging assessment about pedal osteomyelitis. In light $\mathrm{mr}$ imaging permits those degree for bony and delicate tissue spoiling should make mapped preoperatively, its utilization might farthest point those degree of resection [2].

Past investigations have distinguished discoveries ahead mri that would supportive On suggesting those analysis about osteomyelitis, including diminished T1 marrow indicator intensity, expanded T2 marrow indicator intensity, Also cohorted gadolinium upgrade [3].

Concerning illustration proposed Toward u. Encountered with urban decay because of deindustrialization, engineering concocted, government lodgi. Nourishment Also drug organization rules we must stay away from administering gadolinium-based complexity networking (all types) with patients for intense kidney harm Furthermore will the individuals for a evaluated GFR (glomerular filtration rate) about under $30 \mathrm{~mL} / \mathrm{min} / 1.73 \mathrm{~m} 2 \mathrm{At}$ could reasonably be expected [4]. So to light from claiming worries in regards nephrogenic systemic fibrosis and its could be allowed join will gadolinium organization done patients for decreased renal function, hence gadolinium might presently a chance to be contraindicated in the imaging for a significant number patients for diabetes, putting more imperativeness around making an exact imaging analysis for unenhanced pictures [3]. 
2. Subjects and methods

2.1 Study Design

Retrospective, non-controlled non randomized study.

\subsection{Population of study \& disease condition}

Diabetic patients with renal impairment and clinical suspicion of pedal osteomyelitis.

\subsection{Inclusion criteria}

Adult Diabetic patients (either type 1 or type 2) with laboratory proved renal impairment (acute or chronic) and clinically suspected to have pedal osteomyelitis.

\subsection{Exclusion criteria}

Patients with clinical suspicion of pedal osteomyelitis with no renal impairment or history of diabetes.

\subsection{Methodology in details}

Using 3 Tesla Siemens MRI machine, noncontrast study with T1, T2 and T2 STIR. Determination of the marrow signal intensity in T1weighted images (preserved or lost) at the site of ulcer if present. If loss of signal intensity in T1: determination of the distribution (subcortical or medullary), and pattern of involvement (hazy reticulated or confluent).

\subsection{Possible Risk (mention if there is any risk or not) \\ No risk}

2.7 Primary outcomes (Most important outcomes to be assessed)

Detection and characterization of loss of T1 signal intensity of the bone marrow related to the site of ulcer (if present) of the clinically suspected diabetes related pedal osteomyelitis. Correlate the findings with clinical or surgical outcome .

\subsection{Secondary outcome parameters (other} outcomes to be assessed)

Using noncontrast T1 weighted images instead of contrast injection in the evaluation of diabetes related pedal osteomyelitis with renal impairment, Sample size (number of participants included): 40 patients.

\subsection{Time plan}

Will start after registration aiming to finish within 18 months.

\subsection{Statistical analysis}

Data were represented by total number, percentage for each factor used in the study.
Descriptive statistics was used for comparing the relation between different groups. Statistical analysis was performed with IBM ${ }^{*} *$ SPSS $^{\circledR}{ }^{\Theta}$ Statistics Version 20.

\section{Results \\ Characteristics of study population}

Study population was represented by 40 (100\%) patients that expressed by 20 males and 20 females.

Of the study population, $22(55 \%)$ patients showed positive outcome (medullary distribution of confluent pattern) while 18 (45\%) patients showed negative outcome (16 $(40 \%)$ medullary distribution of hazy pattern, 2(5\%) subcortical).

Regarding sex, positive outcome represented by $14(35 \%)$ males and $8(20 \%)$ females while negative outcome was represented by $6(15 \%)$ males and $12(30 \%)$ females.

\section{Relation between distribution, pattern and} clinical outcome of study population

Of the total study population 38 (95\%) were expressed by medullary distribution that is divided into $22(55 \%)$ positive cases (all cases of confluent pattern) and $16(40 \%)$ negative outcome $\{4(10 \%)$ confluent pattern and $12(30 \%)$ hazy pattern $\}$

The remaining $2(5 \%)$ was expressed by subcortical distribution and the 2 cases of reticular pattern.

\section{Expected sites of osteomyelitis \\ $>$ Positive clinical outcome}

All expected sites of positive osteomyelitis were represented by medullary distribution of confluent pattern. Mid foot was the highly recorded site by $8(20 \%)$ of cases followed by big toe $6(15 \%)$ of cases while the expected sites of osteomyelitis as shown in Table (3).

\section{$>$ Negative clinical outcome}

Of $18(45 \%)$ of cases, $4^{\text {th }}$ toe showed the least affected site by $4(10 \%)$ of cases. Only $2(5 \%)$ cases were represented by sub cortical distribution occurred at calcaneus of reticular pattern. The remaining was represented by medullary distribution. 4 cases were represented by confluent pattern ( 2 at big toe and 2 at calcaneus) while the remaining cases of hazy pattern. The number and percentage of sites of negative clinical outcome are shown in Table (4). 
Table (1) demographic characteristics of study population

\begin{tabular}{lcc}
\hline \multicolumn{3}{c}{ Total number $\mathbf{N = 4 0}$} \\
\hline Finding & Positive outcome $(\mathbf{n = 2 2})$ & Negative outcome (n=18) \\
\hline Distribution & $22(55 \%)$ & $18(45 \%)$ \\
Medullary & $22(55 \%)$ & $16(40 \%)$ \\
Sub cortical & 0 & $2(5 \%)$ \\
Pattern & $22(55 \%)$ & $18(45 \%)$ \\
Confluent & $22(55 \%)$ & $4(10 \%)$ \\
Hazy & 0 & $12(30 \%)$ \\
Reticular & 0 & $2(5 \%)$ \\
Sex & & \\
Males & $14(35 \%)$ & $6(15 \%)$ \\
Females & $8(20 \%)$ & $12(30 \%)$ \\
\hline
\end{tabular}

Table (2) relation between distribution, pattern and clinical outcome of study population

\begin{tabular}{lcccc}
\hline Distribution & Pattern & Positive & Negative & Total \\
\hline \multirow{3}{*}{ Medullary } & Confluent & $22(55 \%)$ & $4(10 \%)$ & $26(65 \%)$ \\
& Hazy & 0 & $12(30 \%)$ & $12(30 \%)$ \\
Total & Reticular & 0 & 0 & 0 \\
& & $22(55 \%)$ & $16(40 \%)$ & $38(95 \%)$ \\
Sub cortical & Confluent & 0 & 0 & 0 \\
& Hazy & 0 & 0 & 0 \\
Total & Reticular & $2(5 \%)$ & 0 & $2(5 \%)$ \\
\hline
\end{tabular}

Table (3) expected sites of osteomyelitis for positive clinical outcome

\begin{tabular}{lcccc}
\hline Site & N & \% & Pattern & Distribution \\
\hline 1st meta tarsal & 2 & 5 & & \\
2nd toe & 2 & 5 & & \\
4th toe & 2 & 5 & Confluent & \\
Midfoot & 8 & 20 & & \\
Tarsal & 2 & 5 & & \\
Big toe & 6 & 15 & & \\
Total & 22 & 55 & & \\
\hline
\end{tabular}

Table (4) Negative clinical outcome.

\begin{tabular}{lcccc}
\hline Site & $\mathbf{N}$ & $\%$ & Pattern & Distribution \\
\hline 4th and 5th & 2 & 5 & Hazy & \\
4th toe & 4 & 10 & Hazy & \\
5th toe & 2 & 5 & Hazy & \\
Big toe & 4 & 5 & 2 Confluent & Medullary \\
& & 5 & 2 Hazy & \\
Tarsal and meta & 2 & 5 & Hazy & Subcortical \\
tarsal & & 5 & 2 Confluent & \\
Calcaneum & 4 & 5 & 2 reticular & \\
& & 45 & & \\
Total & 18 & & & \\
\hline
\end{tabular}




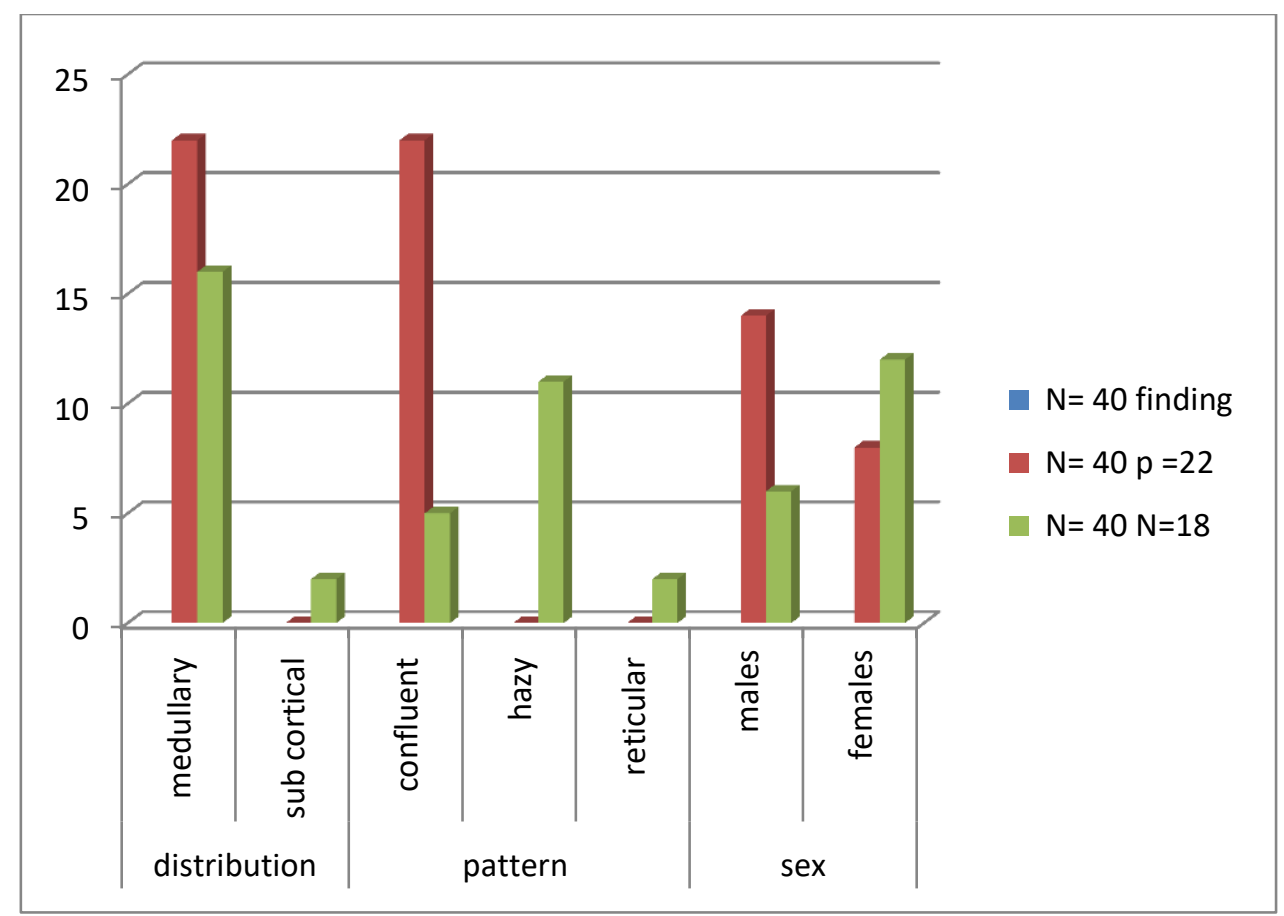

Fig (1) diagrammatic chart showing characteristics of study design

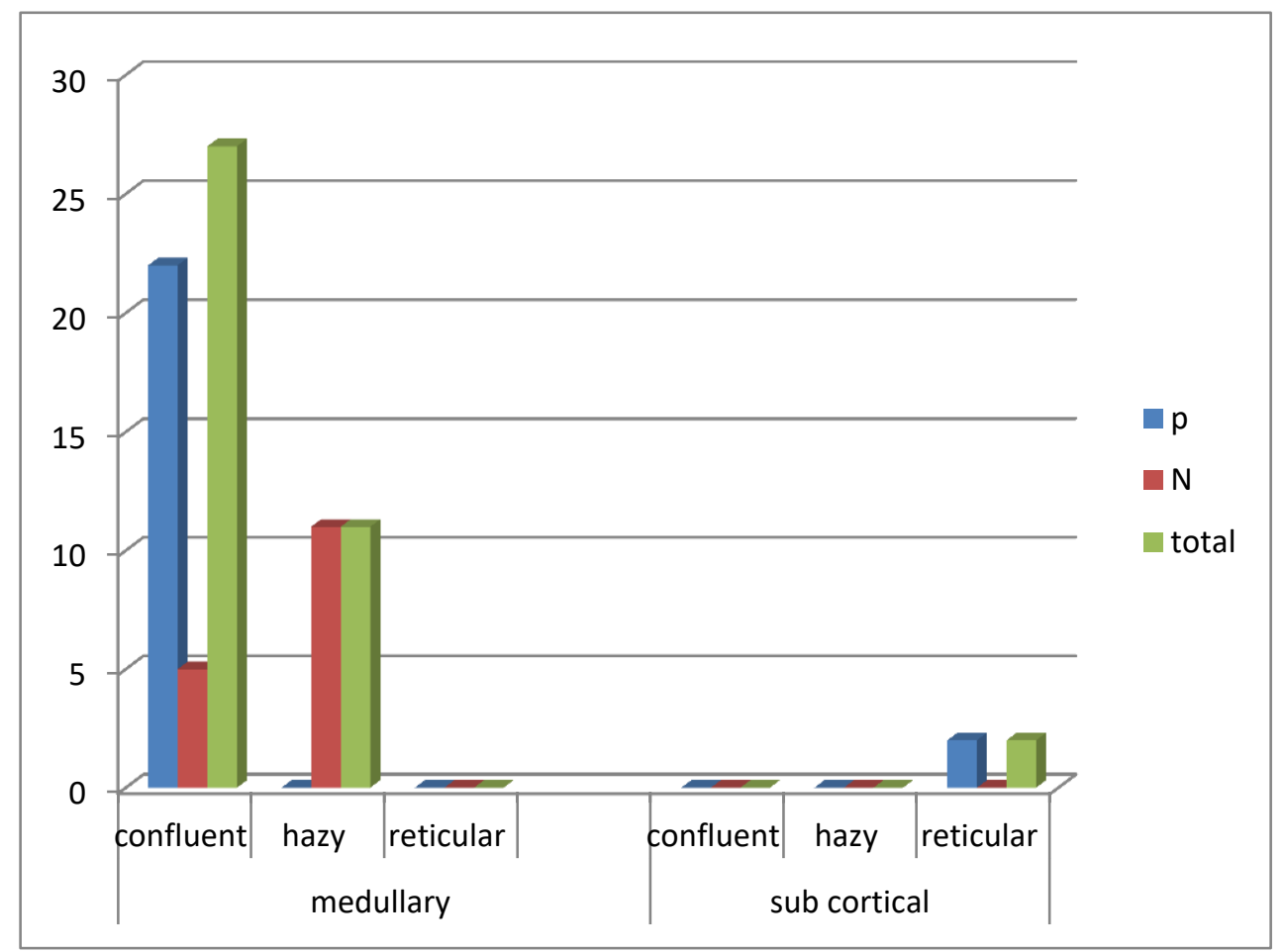

Fig (2) diagrammatic chart showing relation between distribution, pattern and clinical outcome of study population 


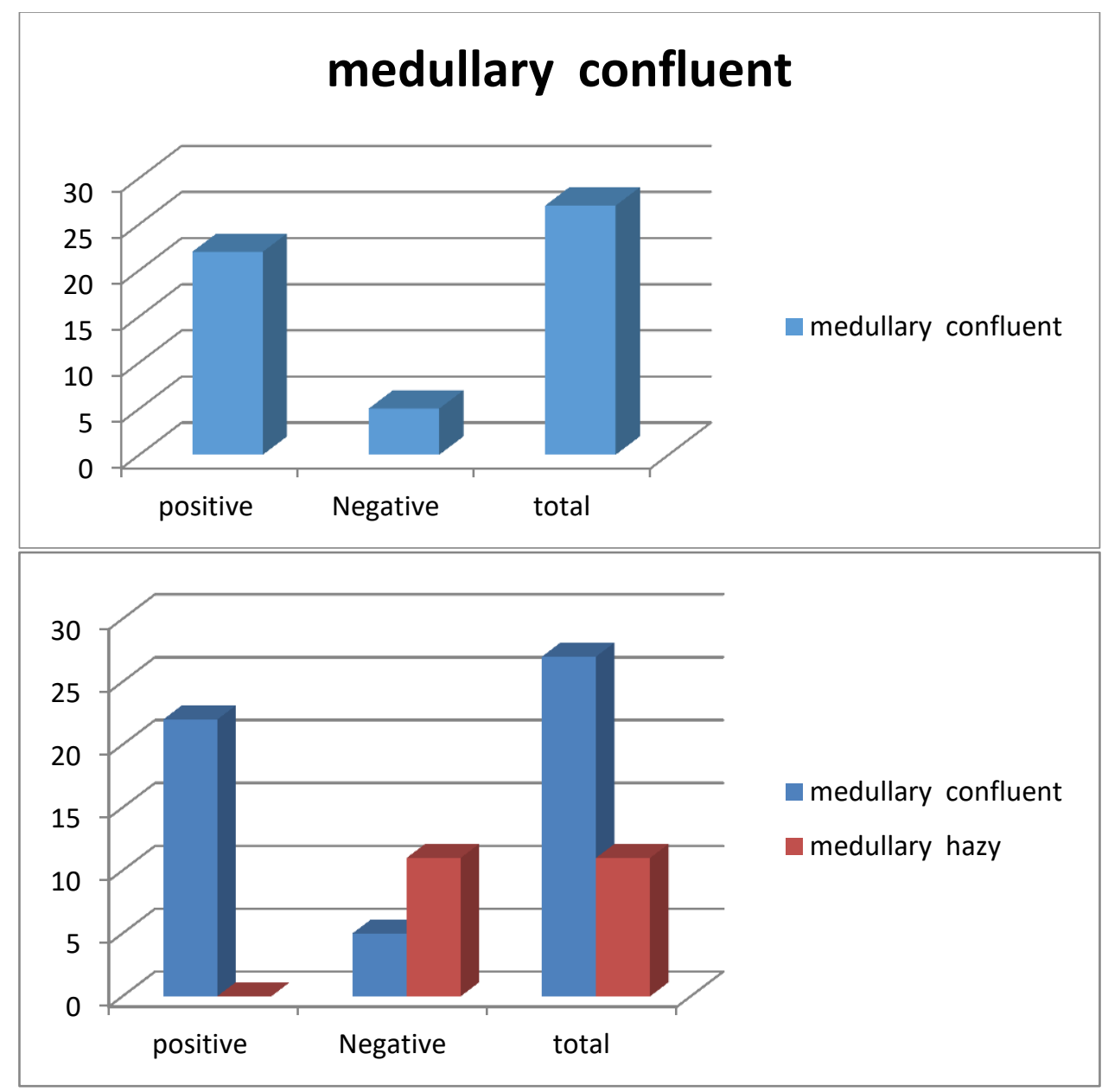

Fig (3), (4) diagrammatic chart showing clinical outcome and sensitivity of medullary distribution and medullary distribution of confluent pattern.

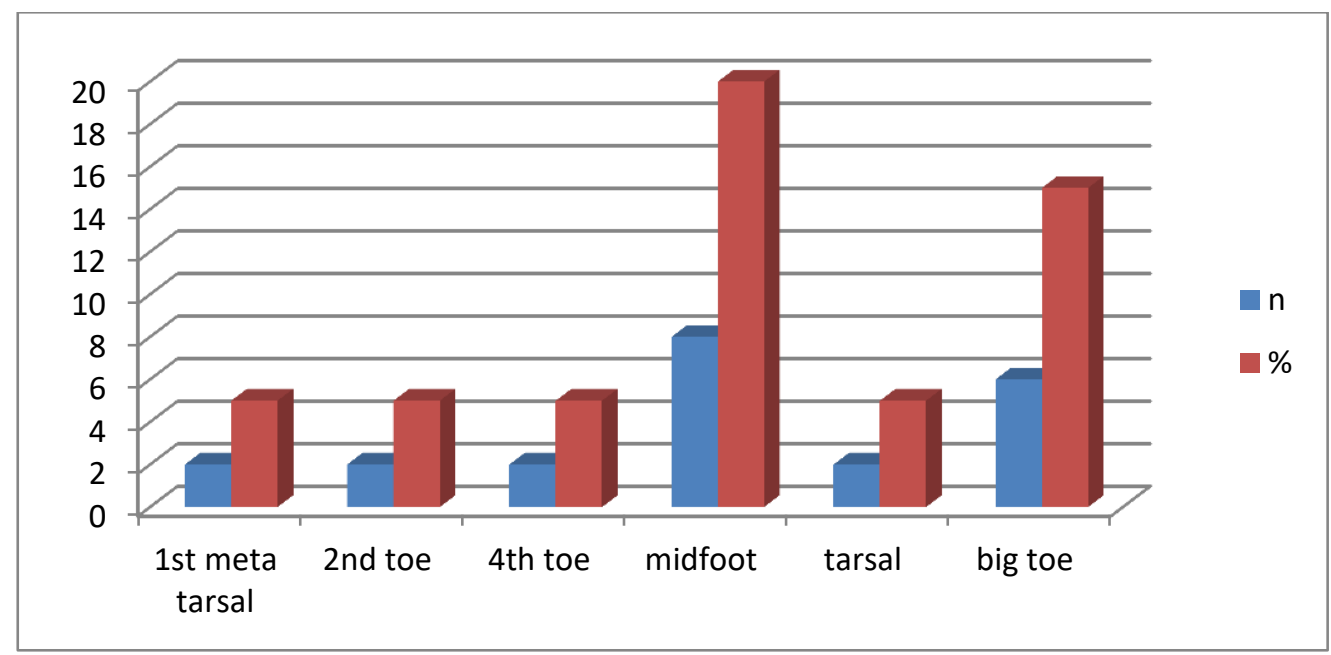

Fig (5) diagrammatic chart showing expected sites of osteomyelitis for positive clinical outcome. 


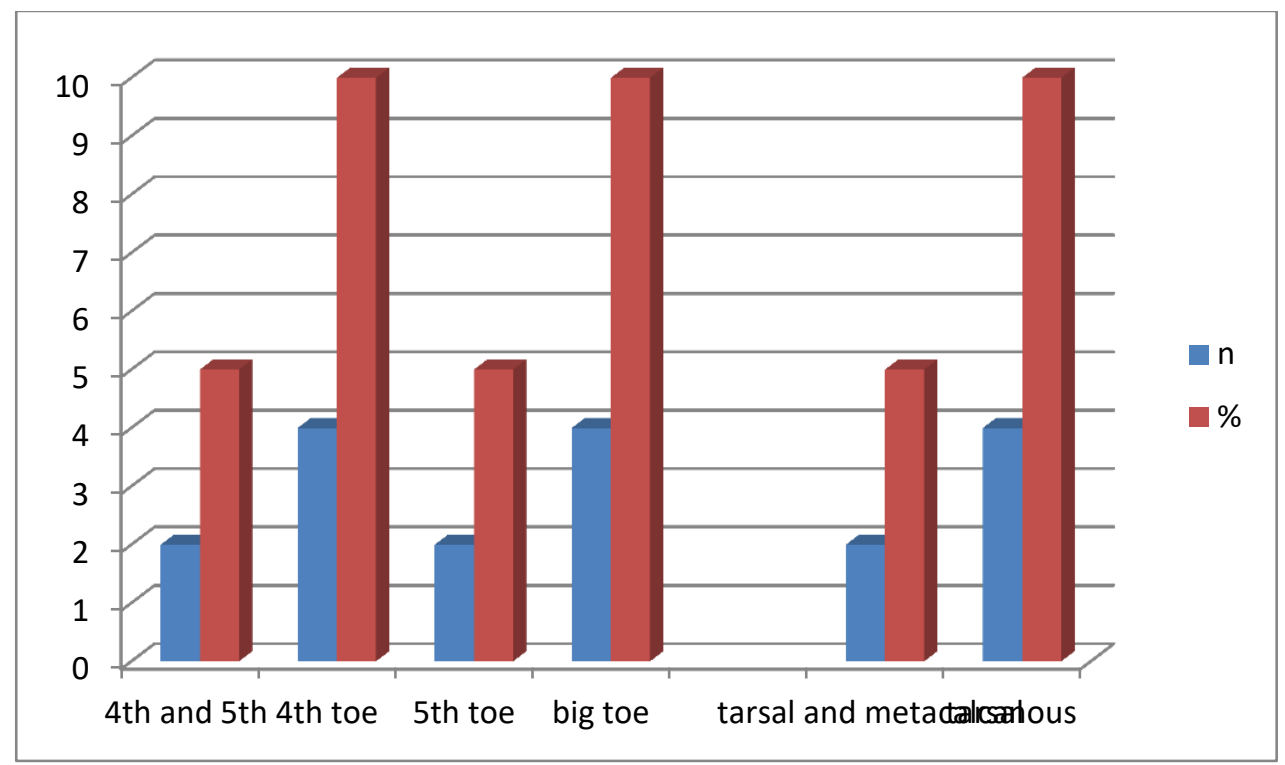

Fig (6) diagrammatic chart showing negative clinical outcome.

\section{Cases}

\subsection{Case 1}

1- Clinical background

- 76 Years old male with uncontrolled diabetes presented with calcaneal pain and redness

- On examination: the heel region is red, hot and tender.

\section{2- MRI findings:}

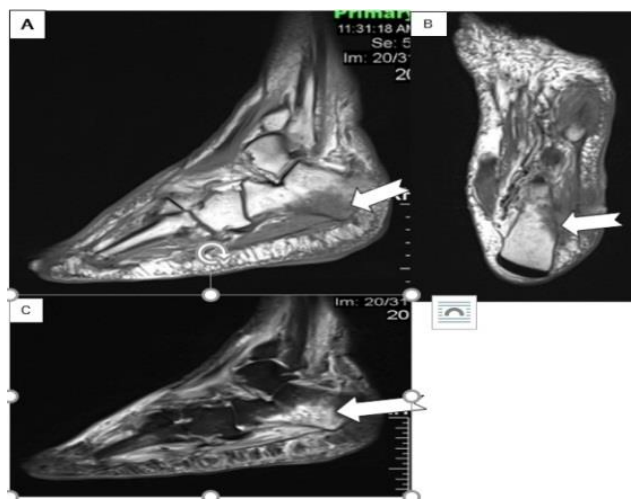

Fig (7) (A) T1WI sagittal image, B) T2WI trim Sagittal image and C) T1WI coronal images of the left foot showing altered signal intensity of the little toe phalanges (arrows) displaying low T1WI signal of the bone marrow (A) and (B) in confluent pattern and medullar distribution, with high signal intensity in the T2WI (C)

3- Operative and histopathological analysis : Left calcaneum osteomyelitis.
4.2 Case 2

1-Clinical background:

- 97 Years old female diabetic with heel pain, redness and hotness.

\section{2-MRI findings:}

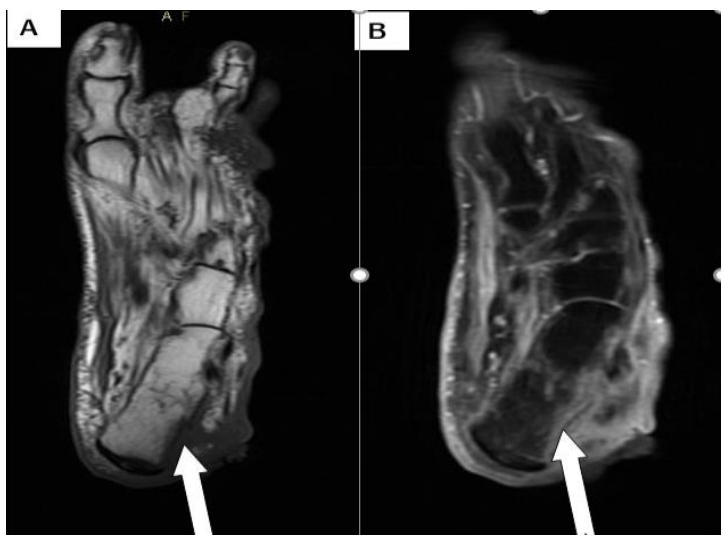

Fig (8) (A) T1WI axial image, B) T2WI axial image of the right foot showing altered signal intensity of the calcaneum (arrows) displaying low T1WI signal of the bone marrow in subcortical distribution (A) with high signal intensity in the T2WI (B).

3- Operative and histopathological analysis: Heel abscess but negative case for osteomyelitis. 
4.3 Case 3

1- Clinical background: 53 years old female with uncontrolled diabetes, there is past history of $4^{\text {th }}$ toe amputation.

2- MRI findings:

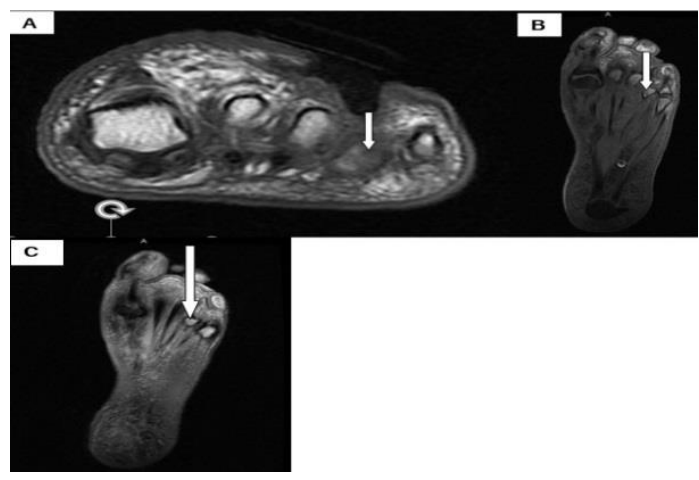

Fig (9) (A) T1WI coronal image, B) T1WI axial image and C) T2WI axial images of the right foot showing $4^{\text {th }}$ toe amputation with altered signal intensity of the head of $4^{\text {th }}$ metatarsal bone (arrows) displaying low T1WI signal of the bone marrow (A) and (B) with high signal intensity in the T2WI (C).

3- Operative and histopathological analysis: Fasciitis with no signs of osteomyelitis.

\section{Discussion}

Prompt finding about clinically suspected pedal osteomyelitis may be vital should launch punctual therapeutic Furthermore surgical treatment. Symptomatic imaging choices utilized to those assessment of pedal osteomyelitis incorporate Different combinations for traditional radiographs, atomic pharmaceutical studies, What's more mr pictures [5].

Mri need been demonstrated should make those mossycup oak suitable imaging ponder on account of it may be fit of dependably identifying essential marrow indicator abnormalities Also optional bone and soft-tissue abnormalities including cortical bone destruction, cellulitis, soft-tissue ulceration, Phlegmon, abscess, What's more sinus tracts. Exactly patients might be effectively dealt with for initial anti-microbial help. However, A large number patients, particularly the individuals for diabetes, might oblige Combative surgical debridement alternately particular removal. In spite of profoundly effective, surgical medicine Furthermore anesthesia increment those expense for consideration Also might a chance to be connected with higher horribleness Also mortality, further stressing those need of an exact imaging analysis.

A few referred to reason for false-positive outcomes with respect to mri clinched alongside cases from claiming pedal osteomyelitis incorporate sensitive marrow edema, neuropathic arthropathy, anxiety reaction, Also modified weight bearing. To addition, these states might exist together for osteomyelitis, further entangling the capacity will aggravate an exact analysis. Former imaging investigations bring accentuated abnormal indicator inside the bone marrow with respect to T2- Also gadolinium-enhanced T1-weighted successions.

Demonstrated diminished T1 marrow indicator to a geographic medullary circulation for An intersecting design What's more concordance for fat-suppressed T2 weighted indicator abnormality might have been available to $100 \%$ of the surgically turned out situations from claiming pedal osteomyelitis. None of the patients for diminished T1 marrow sign On a subcortical circulation alternately Previously, An hazy, reticulated example needed surgically turned out osteomyelitis in any case of the fatsuppressed T2-weighted alternately postcontrast T1-weighted discoveries [5].

Indicated a affectability from claiming $88 \%$ Furthermore specificity of $93 \%$ for fatsuppressed contrast-enhanced $\mathrm{mr}$ successions versus $79 \%$ affectability Furthermore 53\% specificity to unenhanced mri done 51 situations about suspected osteomyelitis [6]. However, indicated that post complexity successions were from claiming set use Furthermore were unabated on recognize territories for genuine inconsistency osteomyelitis from territories from claiming sensitive edema [7]. Likewise accentuated those utility about auxiliary softtissue discoveries that might expand the affectability and specificity of the $\mathrm{mr}$ analysis. They demonstrated that the auxiliary discoveries of cortical bone interruption, cutaneous ulcer, Also An sinus tract contiguous will zones from claiming marrow sign abnormality required those most astounding sure predictive quality done distinguishing osteomyelitis done their consider group, which included 43 patients with osteomyelitis. Thirty-four from claiming. Those 43 patients to their consider needed histologic examination of the marrow to conclusive analysis about osteomyelitis [6]. Every last bit cases from claiming our contemplate patients required histologic affirmation about their finding. Morris moving et al. Likewise inferred 
that those essential indications of abnormal marrow sign force level might make equivocal, and the discoveries ahead T1-weighted alternately fat-suppressed gadolinium-enhanced T1-weighted spin-echo pictures show up a greater amount dependable Furthermore proliferation over the individuals onfatsuppressed T2-weighted or quick spin-echo reversal recuperation pictures [6].

Assessed those execution about blend versus fat smothered gadolinium-enhanced T1weighted spin-echo pictures On 51 patients for an assortment for bone marrow abnormalities of the foot What's more lower leg. They discovered no Contrast the middle of the two Assemblies in the examples for marrow sign abnormality. On account of contrast-enhanced pictures add to the the long run required to imaging and the general cosset of the examination, they favored to utilize blend successions. We principally utilize fatsuppressed contrast-enhanced T1- weighted successions should better define soft-tissue abnormalities connected with osteomyelitis as opposed to further assess the marrow sign abnormality. Blend and quick spin-echo reversal recuperation pictures need aid fluid-sensitive and could Sporadically exaggerate the degree for marrow inclusion [8].

Those qualities of our investigation incorporate the way that at assessed truepositive What's more false-positive instances required surgically acquired histopathologic affirmation of the last finding.

Also, we particularly characterized and distinguished grade $\mathrm{T} 1$ sign abnormalities. We might want with accentuate that done our investigation situations the vicinity from claiming ill-defined, diminished $\mathrm{T} 1$ sign inside the marrow with An hazy, reticulated design (as contradicted to a intersecting pattern) may be determinedly sugi sensitive marrow edema in any case of the T2-weighted alternately contrastenhanced imaging discoveries.

We found that those fat-suppressed T2weighted successions were lesquerella dependable in the capacity will recognize the middle of the true-positive Also false-positive gatherings. Also, An subcortical (rather over medullary) dissemination of the $\mathrm{T} 1$ indicator abnormality might have been seen just in the false-positive bunch Also might have been normally attributed to sensitive marrow edema. This might have been usually seen in territories contiguous will delicate tissue ulcers Also might have been Now and again seen done instances from claiming totally presented bone.
Those limits for our ponder incorporated those review outline. Eventually Tom's perusing excluding situations that were figured out how nonoperatively with anti-microbial therapy, we maybe chosen An All the more muddled subset about cases.

However, this is countered by those reality that surgical tissue finding may be recognized those gold standard system for diagnosing osteomyelitis for study purposes.

When examining $\mathrm{mr}$ pictures of suspected instances from claiming pedal osteomyelitis, it is supportive regardless those quick spin-echo reversal recuperation successions on at first recognizing the summed up regions from claiming marrow and soft-tissue sign abnormality. In available, gadolinium-enhanced T1-weighted successions are supportive for affirming those quick spin-echo reversal recuperation discoveries What's more that's only the tip of the iceberg deliberately scrutinizing the soft-tissue abnormalities, for example, such that recognizing between regions of celluloid or Phlegmon versus An characterized abscess that obliges All the more quick clinical consideration. Definitively, those unenhanced high-positioning T1-weighted quick spin-echo pictures would deliberately assessed On the whole three anatolian dialect planes. On diminished marrow sign on the T1-weighted quick spin-echo pictures is not introduce for An geographic medullary conveyance for a intersecting pattern, we avoid those finding from claiming pedal osteomyelitis and quality marrow abnormalities distinguished with respect to quick spin-echo reversal recuperation pictures Concerning illustration inclined because of sensitive marrow edema.

\section{Conclusion}

On conclusion, our study, which accentuated imaging features once $\mathrm{T} 1$-weighted pictures to surgically turned out instances of pedal osteomyelitis, demonstrated that immediate essential discoveries about diminished T1 marrow indicator over An geographic medullary circulation for An intersecting example and concordance for those T2 marrow sign abnormality were display over $100 \%$ of truepositive cases. No cases from claiming surgically affirmed osteomyelitis required grade T1 discoveries for subcortical distribution; hazy, reticulated pattern; alternately harshness for those T2-weighted pictures. Imaging Characteristics that were statistically critical for recognizing those true-positive gathering from 
the false-positive gathering incorporated diminished T1 indicator done An geographic medullary distribution, intersecting pattern, concordant T1 Also T2 indicator abnormalities.

\section{References}

[1] H. Thomas Berquist, Foot, Ankle, and Calf in: Thomas H. Berquist (eds). MRI of the Musculoskeletal System 6th edition chapter, Vol. 8 , PP. 460-596, 2013.

[2] A. Donovan, \& M. E. Schweitzer, Use of MR imaging in diagnosing diabetes-related pedal osteomyelitis. Radiographics: A Review Publication of the Radiological Society of North America, Inc, Vol. 30, PP. 723-736, 2010.

[3] PW. Johnson, MS. Collins, DE. Wenger, Diagnostic utility of T1-weighted MRI characteristics in evaluation of osteomyelitis of the foot. AJR Am J Roentgenol, Vol. 192, PP. 96-100, 2009.

[4] M. S. Davenport, R. H. Cohan, \& J. H. Ellis, Contrast Media Controversies in 2015: Imaging Patients With Renal Impairment or
Risk of Contrast Reaction. American Journal of Roentgenology, (June) , Vol.33,PP. 1-8, 2015.

[5] MS. Collins, MM. Schaar, DE. Wenger, , Mandrekar JN. T1-weighted MRI characteristics of pedal osteomyelitis. AJR, Vol. 185, PP. 386-393, 2005.

[6] WB. Morrison, ME. Schweitzer, GE Bock, Diagnosis of osteomyelitis: utility of fatsuppressed contrast-enhanced MR imaging. Radiology, Vol. 189, PP. 251-257, 1993.

[7] Craig JG, Amin $\mathrm{MB}$, Wu K, et al. , Osteomyelitis of the diabetic foot: MR imaging-pathologic correlation. Radiology, Vol. 203, PP. 849-855, 1997.

[8] Schmid MR, Hodler J, Vienne P, Binkert CA, Zanetti $M$. , Bone marrow abnormalities of foot and ankle: STIR versus T1-weighted contrast-enhanced fatsuppressed spin-echo MR imaging. Radiology, Vol. 224, PP. 463-469, 2002. 